\title{
Pilot study of revised stillbirth and neonatal death certificates
}

\author{
B GEDALLA AND M R ALDERSON \\ Office of Population Censuses and Surveys, London
}

SUMMARY Draft revised stillbirth and early infant death certificates were sent with a short questionnaire to certifying doctors for 206 stillbirths and 198 neonatal deaths for which death certificates had been received in the Office of Population Censuses and Surveys over a three week period in 1982. The new certificates complied with the recommendations of the World Health Organisation and asked for details of the cause of death, including separate sections for maternal and fetal conditions.

Replies were received for 163 stillbirths and 151 neonatal deaths. Questionnaires were examined clerically and answers and comments analysed. Draft certificates were processed by computer to examine any differences between the causes of death given on the new forms and information given on the original certificates. This testing suggested that revised certificates are acceptable. Extension of the early infant death certificate to cover all neonatal deaths does not seem to create problems and should provide information on the association between maternal conditions and deaths occurring in the second to fourth weeks of life.

If the full potential of the new certificates is to be realised, attention needs to be given to the detailed layout of the forms and clearer notes of guidance. Certifiers need to be encouraged to give appropriate details of any obstetric problems.

Registration of death was introduced in England and Wales in 1837 and since then information on causes of death has been collected, firstly by the General Register Office and, since 1970 , by its successor the Office of Population Censuses and Surveys (OPCS). Until 1926, however, there was no requirement to register stillbirths. The Births and Deaths Registration Act of 1926 introduced compulsory registration for stillbirths (defined as 'a child which has issued forth from its mother after the twenty-eighth week of pregnancy and which did not at any time after being completely expelled from its mother breathe or show any other signs of life') but did not provide for collection of cause of death information. Only in 1960, with the Population (Statistics) Act of that year, was cause of death certification introduced for stillbirths. (The same act required stillbirth certification to be performed by a medical practitioner, or if no such person at any time examined the child either during or after delivery, a midwife; before this most stillbirths were registered by midwives irrespective of whether or not a doctor had attended the delivery.)

Thus, the history of the collection of cause of death statistics for stillbirths and deaths is quite separate and although the formats used for the collection of this information (reproduced in Fig. 1 (stillbirth) and Fig. 2 (death)) are similar, the notes for guidance issued with them are quite different. When studying perinatal and neonatal mortality this disparity can be unfortunate since many early infant deaths are caused by sequences of events that have more similarity to those occurring in stillbirths than in adult deaths. Further, although the stillbirth certificate in current use (Fig. 1) does indicate the need by certifying doctors to mention conditions arising in the mother as well as those arising in the fetus, no provision is made for those events in which the doctor thinks that both maternal and fetal conditions contributed equally to the death. For early infant deaths the current notes for guidance make only passing mention of maternal factors and information on maternal factors is infrequently provided by the certifiers.

Because of the problems in satisfactorily identifying maternal causes in stillbirths and early infant deaths the Brodrick Committee ${ }^{1}$ and subsequently the World Health Organisation ${ }^{2}$ have advocated 


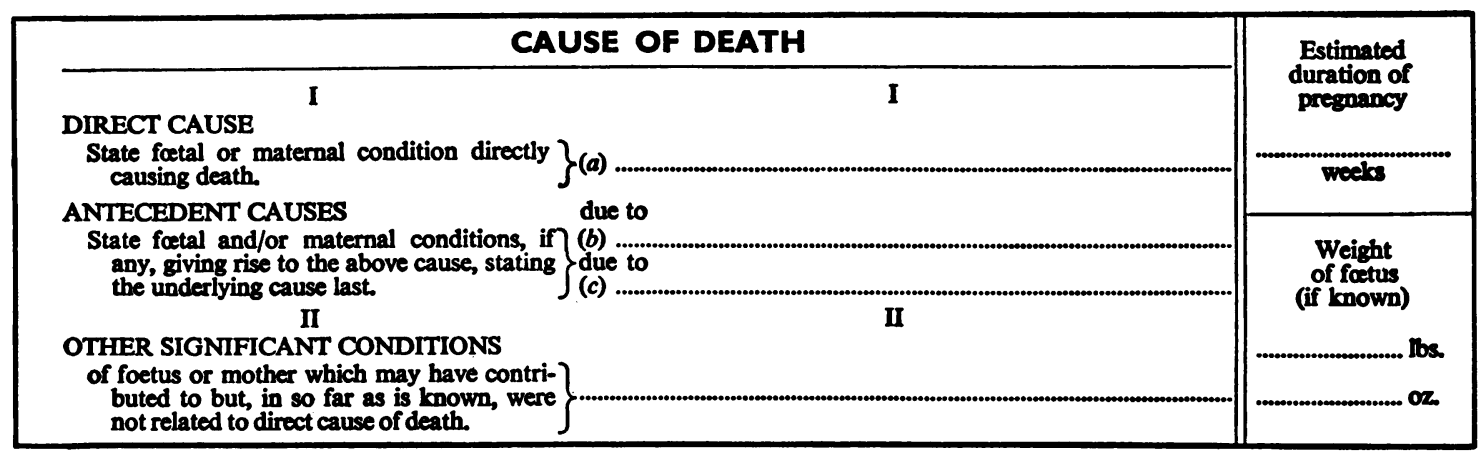

Fig. 1 Cause of death information requested on existing stillbirth certificate.

\section{CAUSE OF DEATH}

Disease or condition directly leading to death†

$\left.\begin{array}{l}\text { Antecedent causes. } \\ \text { Morbid conditions, if any, giving rise } \\ \text { to the above cause stating the }\end{array}\right\}$

underlying condition last.

II

Other significant conditions,

contributing to the death, but not related

to the disease or condition causing it. that deaths in the perinatal period (that is, stillbirths and deaths in the first week of life) should be certified using forms that request completion of particulars about both fetal and maternal conditions. Further support has come from the Royal College of Obstetricians and Gynaecologists ${ }^{3}$ and the House of Commons Select Committee on the Social Services. ${ }^{4}$ Reports have already been published of the use of these certificates in New Zealand $^{5}$ and Northern Ireland. ${ }^{6}$

This report is based on a pilot study in England and Wales of new forms for certifying stillbirths and early infant deaths. The new forms were designed to take into account the recommendations described above. The pilot study had two rather different aims; firstly to determine whether the forms were acceptable to certifiers, and secondly to ascertain whether the statistics provided were more useful than data available from the present forms. There have recently been suggestions ${ }^{78}$ that advances in medical care of the newborn are postponing some early infant deaths into the late neonatal period (that is, up to age 4 weeks). To take account of this possibility, the pilot study was extended to include deaths throughout the neonatal period (rather than just the first week of life) and thus one particular aspect of acceptability was to see whether this extension would meet with the doctors' approval.

\section{Method}

A sample was drawn from all certificates of stillbirths and deaths in the first four weeks, occurring in hospitals, and which reached OPCS in the three weeks beginning 27 September 1982. Deaths at home certified by general practitioners or coroners were excluded from the study (these are so rare in this age group that deaths for an entire year would not have produced an adequately sized sample).

A letter signed by one of us (MRA) was sent to the certifier about two weeks after the stillbirth was delivered or the neonatal death occurred. This was attached to a new draft form to be completed together with a brief questionnaire and a copy of the original stillbirth or death certificate. Fig. 3 shows the new format of the cause of death section. If no response had been received after three weeks a reminder letter was sent, again signed by MRA.

The study was processed in two stages. As the forms were received, the questionnaires were sep- 


\begin{tabular}{|c|c|}
\hline & CAUSE OF STILLBIRTH [DEATH] \\
\hline & $\begin{array}{l}\text { Main disease or condition in fetus [infant ] } \\
\text { Other diseases or conditions in fetus [infant] * }\end{array}$ \\
\hline c & $\begin{array}{l}\text { Main maternal disease (or condition) affecting fetus } \\
\text { [infant ]* }\end{array}$ \\
\hline d & $\begin{array}{l}\text { Other maternal diseases (or conditions) affecting fetus } \\
\text { [infant ]* }\end{array}$ \\
\hline e & Other relevant causes \\
\hline
\end{tabular}

Fig. 3 Cause of death section of forms used in the pilot study.

* Indicates alternative wording for stillbirth and first 4 week death.

arated from the draft certificates, were examined clerically, and an analysis of the answers and comments was produced. The details of this analysis are discussed in the Results section. The second stage of processing was the more formal analysis of the causes of death given on both old and new certificates. The method used by the coders was as follows: for all events included in the study the original draft entry was coded for causes(s) of death using the normal (ICD 9th revision) coding rules, producing an underlying cause together with up to eight causes. For those for which a reply was received by the closing date, the new forms were coded to produce again up to eight causes with the principal cause in box A (main condition affecting fetus) taken to be the main fetal cause and the principal cause in box $\mathrm{C}$ (main condition affecting mother) taken to be the main maternal cause. Details of the analysis of the results are discussed below.

\section{Results}

The total number of certificates of stillbirths and early infant deaths received in the three week period was 404 (206 stillbirths and 198 neonatal deaths). By the closing date (some six weeks after the last death certificates were selected), 314 replies $(78 \%)$ had been received. The response rates were similar in both categories with 163 stillbirth forms $(79 \%)$ and 151 neonatal death forms $(76 \%)$ being returned by the certifying doctors. Bearing in mind that the certifiers will have included an appreciable proportion of junior doctors who may only be in post for six months, this reponse is very satisfactory.

Questionnaires. Respondents replies to the questionnaires are presented below, firstly for stillbirths and secondly for neonatal deaths.

\section{Stillbirth certificates}

(1) Were there specific problems in completing the new form? Thirty eight of the 163 respondents indicated that there had been problems but 26 of these were either generated because of the pilot form (which would be avoided were the new form to be introduced for routine use) or were suggesting alterations in layout of the form which will be taken into account if the new form is introduced. Eight stated that the cause of death was not precisely determined and this created a problem in certification. In five there was some doubt as to the appropriate place to enter some of the diagnostic information, and one was a technical query about the difficulty of certifying cause of death in major congenital malformation.

(2) Has further information become available since you completed the initial certificate? In 74 of the deaths further information had become available after the initial stillbirth certificate was completed (in 63 of these after necropsy, in six after perinatal death review, and in five from other information).

(3) Are there any general comments you wish to make about this topic? Thirty six respondents provided comments but only one of these was a suggestion that the current form should not be changed. Four drew attention to the general problems in determining the cause of stillbirth, and others made remarks such as the delay in obtaining a necropsy report, the desirability of completing the form only after necropsy, and the need for other investigations to be carried out-particularly in congenital malformation. Six responses were specifically in favour of the new form.

\section{Early infant death certificate}

(1) Were there specific problems in completing the new form? Twenty five respondents recorded comments, but none suggested that the new form should not be used in general. The points made were of the kind one would have anticipated: five had difficulty in identifying the age of the deceased in hours, in three maternal details were not readily available, and in 11 there were problems in identifying the cause. One respondent criticised the lack of ranking on the new form (the intention is deliberately not to rank the items, but request material relating to different aspects of the cause of death under different headings). Two thought the amount of information required was excessive.

(2) Has further information become available since you completed the initial certificate? In 83 subjects further information had become available; from 
necropsy in 71, from perinatal death review in four, and from other sources in eight.

(3) Are there any general comments you wish to make about this topic? Forty seven of the respondents provided specific comments. Sixteen seemed to welcome the new form, though three others asked for more information to guide them in completing it. Again problems in determining the cause of death were identified by eight, one mentioned delay in obtaining necropsy information, and one difficulty in obtaining maternal information when an infant was transferred to paediatric care. There were other suggestions which are not directly relevant (for example six suggesting birthweight should be entered (when this is already obtained through different mechanisms) others requested gestational age at birth, which legal opinion states cannot be included under present law).

Cause of death analysis. Analyses were conducted comparing the underlying causes of death derived from the old certificates with the main fetal and maternal causes derived from the new forms. In general, the (new) fetal causes were similar to the (old) underlying causes for both stillbirths and early infant deaths. There were only 10 (all stillbirths, representing six per cent of the completed stillbirth test forms received) in which only maternal causes were given. In contrast, in 100 stillbirth forms and 94 neonatal death forms (in each case, $63 \%$ of the forms returned) no maternal causes were stated. In the remainder a wide variety of maternal conditions were given and these are summarised in Table 1. Full tabular analyses comparing the causes given in the original certificates and those in the new forms were prepared showing the (old) underlying causes and the (new) fetal causes aggregated into the main groups of causes usually shown in published tables, with the (new) maternal causes shown explicitly, as in Table 1 . These tables are fairly long and have many cells containing very small numbers (owing to the small size of the test sample). For the sake of brevity they are not included here, but are available on request from the authors.

It is expected that routine mortality statistics derived from the new forms in actual use would show cross analyses of fetal cause by maternal cause. The sample size used for this study was too small to generate sufficiently useful statistics to merit such tabulations being produced.

Table 2 shows proportions of deaths in which the fetal cause on the new certificate was not in the same broad category as the underlying cause in the original certificate. Almost $70 \%$ of all responses remained in the same category. Within specific

Table 1 Maternal causes reported on the new stillbirth and neonatal death certificates

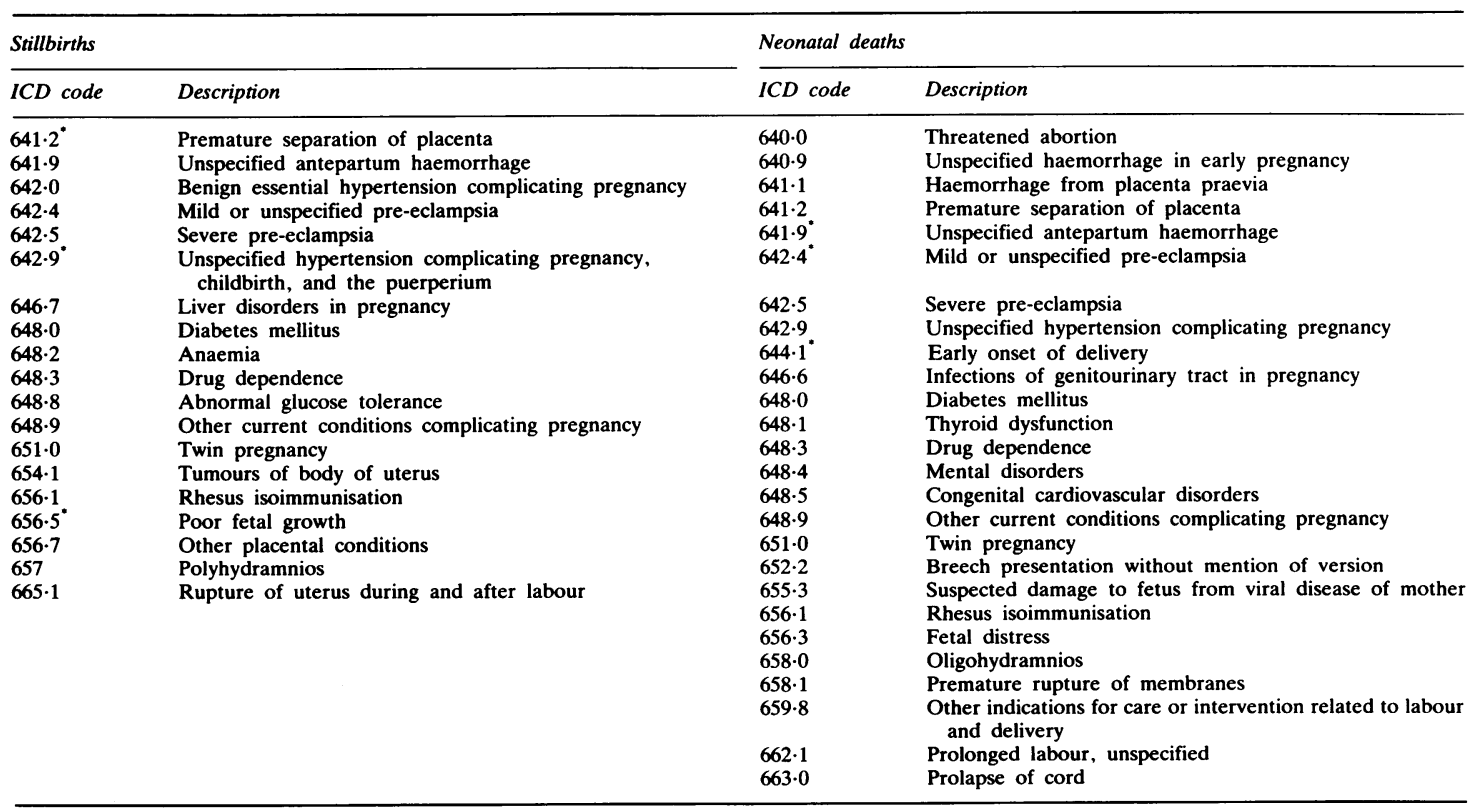

Denotes the three most commonly stated conditions. 
Table 2 Proportion of deaths in which the fetal cause on the new certificate was in the same category as the underlying cause on the original certificate

\begin{tabular}{|c|c|c|c|}
\hline \multirow{2}{*}{$\begin{array}{l}\begin{array}{l}\text { Original } \\
\text { underlying cause }\end{array} \\
\text { Respiratory causes }\end{array}$} & $\begin{array}{l}\text { No of stillbirths } \\
\text { or deaths }\end{array}$ & \multicolumn{2}{|c|}{$\begin{array}{l}\text { Fetal cause the same as the } \\
\text { original underlying cause }\end{array}$} \\
\hline & & & \\
\hline Stillbirths & - & - & - \\
\hline Neonatal deaths & 2 & 2 & $(100)$ \\
\hline Total & 2 & 2 & $(100)$ \\
\hline \multicolumn{4}{|c|}{ Congenital malformations } \\
\hline Stillbirths & 21 & 19 & $(90 \cdot 5)$ \\
\hline Neonatal deaths & 56 & 42 & $(75 \cdot 0)$ \\
\hline Total & 77 & 61 & $(79 \cdot 2)$ \\
\hline \multicolumn{4}{|c|}{ Obstetric complications } \\
\hline Stillbirths & 64 & 27 & $(42 \cdot 2)$ \\
\hline Neonatal deaths & 6 & - & $(0 \cdot 0)$ \\
\hline Total & 70 & 27 & $(38 \cdot 6)$ \\
\hline \multicolumn{4}{|l|}{ Fetal immaturity } \\
\hline Stillbirths & 3 & 1 & $(33 \cdot 3)$ \\
\hline Neonatal deaths & 18 & 17 & $(94 \cdot 4)$ \\
\hline Total & 21 & 18 & $(85 \cdot 7)$ \\
\hline \multicolumn{4}{|l|}{ Hypoxia, etc } \\
\hline Stillbirths & 21 & 19 & $(90 \cdot 5)$ \\
\hline Neonatal deaths & 46 & 32 & $(69 \cdot 6)$ \\
\hline Total & 67 & 51 & $(76 \cdot 1)$ \\
\hline \multicolumn{4}{|c|}{ Other perinatal causes } \\
\hline Stillbirths & 48 & 35 & $(72 \cdot 9)$ \\
\hline Neonatal deaths & 17 & 12 & $(70 \cdot 6)$ \\
\hline Total & 65 & 47 & $(72 \cdot 3)$ \\
\hline \multicolumn{4}{|l|}{ Other causes } \\
\hline Stillbirths & 1 & - & $(0 \cdot 0)$ \\
\hline Neonatal deaths & 6 & 3 & $(50 \cdot 0)$ \\
\hline Total & 7 & 3 & $(42 \cdot 9)$ \\
\hline \multicolumn{4}{|l|}{ Total } \\
\hline Stillbirths & 158 & 101 & $(63 \cdot 0)$ \\
\hline Neonatal deaths & 151 & 108 & $(71 \cdot 5)$ \\
\hline Overall & 309 & 209 & $(67 \cdot 6)$ \\
\hline
\end{tabular}

causes many of the percentages are higher, with three being over $90 \%$. The principal exception to this was deaths originally certified as caused by obstetric complications. That this should be so is perhaps not too surprising since the new form encourages the doctor to give a cause more specific to the child and this is reflected in the results-28 of the stillbirths that have changed category have a new fetal cause of hypoxia or another cause originating in the perinatal period. Indeed, only seven of the stillbirths in this category were given only maternal causes on the new form (all seven were coded 641.2 Premature separation of placenta).

A computer listing was produced of those deaths in which the (old) underlying cause differed from the (new) fetal cause. The listing showed all the multiple causes assigned to these deaths from both forms. The list was examined to identify those where the new fetal cause was in a completely different category (using the categories in Table 2) from the old underlying cause and where the old underlying cause was not given at all in the list of new causes.
This procedure identified 43 stillbirths and 17 neonatal deaths in which a major change seemed to have occurred. Both forms were extracted for these 60 and a more detailed examination undertaken, the results of which are shown in Table 3.

Table 3 shows that 25 of the 43 stillbirths had an (old) underlying cause of placenta abruptio or placental insufficiency - causes in the obstetric complications category discussed above. In all these the placental problem was originally assigned an ICD code relating to the child, while the position of the same condition on the new form had resulted in it being coded as a maternal condition. Thus, apart from new forms in which causes of death were changed by the doctor as a result of further information (for example necropsy) being available to him, in the vast majority the basic fetal cause of death given in the original certificate was the same

Table 3 Analysis of cases where old underlying cause code was not assigned at all on the new form

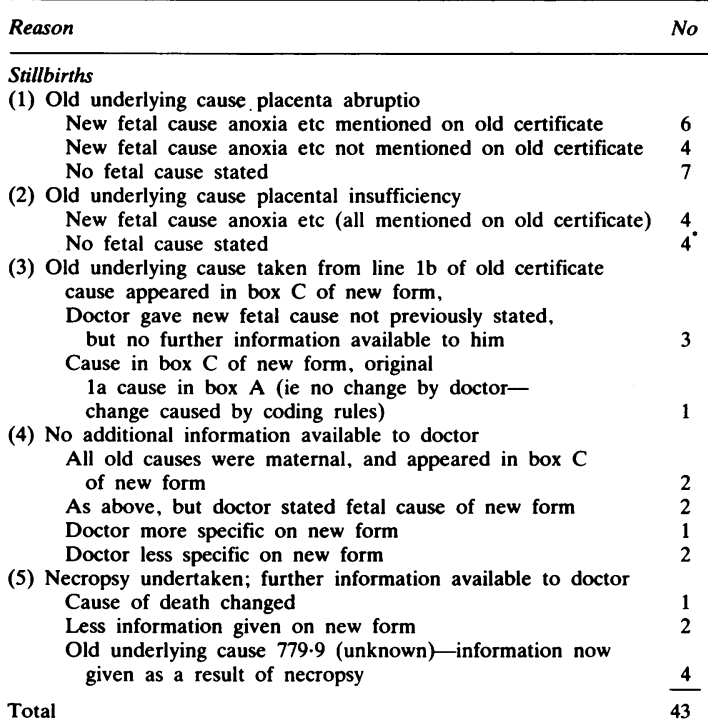

Neonatal deaths

(1) Old underlying cause taken from line 1b of old certificate Original la cause in box $A$ (ie no change by doctor-change caused by coding rules)

Original 1a cause in box $A$ but extra information also given by doctor

(2) Old underlying cause, taken from line 2 of old certificate $\quad 3^{\dagger}$ Order of causes reversed by doctor

(3) No additional information available to doctor Original 1a cause omitted on new form but additional maternal information given Less information given on new form (5) Necropsy undertaken; causes changed Total

"One case new form completed as 'nil'; 'two cases were twins. 
in the new. It would, therefore, seem that the new forms did not impair the information given by the doctors (apart, that is, from a very small number of deaths where the doctors gave less information on the new form than on the original) and it may be safely concluded that the introduction of this type of form will not have a detrimental effect on the data collected.

The purpose of this exercise, however, was to ascertain whether the new form would increase the information collected. To this end, two more listings were produced, to show all causes (from both old and new forms) for those events where (a) the new main fetal cause code and (b) the new main maternal cause code were not included at all in the original list of causes assigned from the original certificate. This method was chosen because in the past only limited use has been made of multiple cause information and it seems reasonable to assume that most analyses of cause of death in the future, based on the new certificates, would also concentrate on the main causes (either fetal or maternal, or both). It was hoped to show whether the information obtainable from the two main causes given on the new form could have been procured by detailed examination of the causes given on the original certificate. The listings were examined to eliminate those events where the main cause differed only slightly from one of the original causes (that is, where the discrepancy was a result of a very slight change in terminology). Also eliminated were those events where the new cause code related to a condition included in the original list, which had initially been reported as a fetal condition but in the new form had been identified as a maternal condition (for example, maternal hypertension).

The conclusion of this analysis was that, broadly speaking, the amount of information gained was only slightly increased by the new forms. The results of the analysis of the listing of events where the new fetal cause had not previously been given were not too surprising. There were just 12 stillbirths where the new fetal cause had not been included on the original certificate and in seven of these the new data were given as a result of further information (necropsy, perinatal death review) being available to the doctor when the new form was completed. For neonatal deaths, there were only 16 examples of a different main fetal cause, 13 of which were due to additional information.

Examination of the listing of stillbirths where the new main maternal cause code had not previously been given showed that there were just six (three as a result of further information) where the maternal cause was genuinely not shown on the original certificate. For neonatal deaths the results were a little more encouraging with 36 examples of new maternal information (18 due to further information).

Setting aside those deaths where the additional information could be attributed to necropsy or other post mortem investigation, there were only 29 events $(7 \%)$ where the new certificates yielded more information about the main cause of death. It has been suggested, however, that the introduction of the new certificate should be coupled with a substantial increase in the number of instances where OPCS submits a medical enquiry to the consultant responsible for the care of the mother or child; this should ensure that information is made available after a necropsy in a larger number of cases. Completion of such an enquiry by a more senior doctor than the certifier, particularly after a 'perinatal audit' meeting has discussed all available information, might lead to provision of additional maternal and fetal conditions. ${ }^{9}$ Although the present study only indicates that further information may be available from some $17 \%$ of cases (based on the 70 in the study), the pilot may not reflect the additional effect of involving a more senior doctor and discussion from the perinatal audit. This view has been supported by experience in Scotland (Walker CHM, personal communication).

We thank the certifying doctors who took the time and trouble to complete the test forms. We would also like to thank the several colleagues whose comments and advice were received during the preparation of this paper.

\section{References}

${ }^{1}$ Report of the Committee on Death Certification and Coroners. Cmnd 4810. London: HMSO, 1971.

2 World Health Organisation. International classification of diseases, 9th revision, vol 1. Geneva: WHO, 1977.

${ }^{3}$ Scott MJ, Ritchie JWK. A study of the World Health Organisation perinatal death certificate in Northern Ireland in audit and perinatal surveillance. Proceedings of the eighth study group of the Royal College of Obstetricians and Gynaecologists, 1980.

${ }^{4}$ Third Report of House of Commons Select Committee on the Social Services. Perinatal and neonatal mortality. Cmnd 663-1. London: HMSO, 1980.

5 World Health Organisation. A WHO report on social and biological effects on perinatal mortality. Vol I. Geneva: WHO, 1978:152.

${ }^{6}$ Scott MJ, Ritchie JWK, McClure BG, McCreid M, Halliday $\mathrm{HL}$. Perinatal death recording: time for a change? $\mathrm{Br}$ Med $J$ 1981;282:707-10.

7 Arneil GC, Brooke H, Gibson AMM. Post-perinatal infant mortality in Glasgow 1979-1981. Lancet 1982;ii:649-51.

${ }^{8}$ Macfarlane AJ. A time to die? Int J Epidemiol 1984;13:38-44.

9 Joint Report of the Royal College of Physicians and Royal College of Pathologists. Medical aspects of death certification. J R Coll Physicians Lond 1982;16:202-18.

Correspondence to Mr B Gedalla, Medical Statistics Division, OPCS, St Catherine's House, 10 Kingsway, London WC2B 6JP.

Received 14 June 1984 